\title{
Diffraction from Oxide Superlattices - Stacking Sequence Effects in Plan View.
}

\author{
J.C.H. Spence, A. B. Shah, U. Weierstall, B.B. Nelson-Cheeseman * and A. Bhattacharya*. \\ Department of Physics, Arizona State University, Tempe, AZ 85287-1504 \\ *Materials Science Division, Argonne National Laboratory, Argonne, IL 60439
}

As nanoscience moves from observation to control, and electron microscope image interpretation becomes simplified by the new corrected TEMs, there is now a more realistic prospect than ever of both fabricating, with control at the atomic level, structures with wanted properties, and interpreting their bulk properties in terms of the atomic structures seen in the (S)TEM. Oxide superlattices, for example, may now be tailor-made by atomic-layer deposition to produce both conductive and ferromagnetic or antiferromagnetic interfaces by choice of stoichiometry and stacking sequence [1].

"Termination" or forbidden Bragg reflections (such as the (-422)/3 in silicon) have been used to image stacking faults in single-beam dark field TEM mode [2], since they are generated only by this additional plane of atoms in a thin sample with atomically rough surfaces. (Sharp termination can also generate these spots under UHV conditions). The perovskite structure, popular for digital oxide superlattices, has no such termination reflections, however the "difference" reflection $(1,0,0)$ is extremely sensitive to stacking sequence, as shown in figure 1 . Here we show, with incident beam [001] normal to interfaces, the calculated intensity of the (100) reflection from the superlattice shown in figure 2 [3], compared with that from the ideal LaMnO3 structure. The superlattice contains an offset layer of $\mathrm{SrMnO} 3 .(\mathrm{A}=\mathrm{LaO}$ layer, $\mathrm{a}=\mathrm{MnO} 2$ layer, $\mathrm{B}=\mathrm{SrO}$ layer, prime indicates $a_{o} / 2$ shear along [100]). The upper curve does not contain the sheared layer whose interfaces $\mathrm{AB}^{\prime}$ and B'A may be responsible for observed ferromagnetism [1]. Plan view TEM single-beam darkfield images using this reflection would thus show large contrast differences between sheared and unsheared regions, and, if quantified, could provide a useful measure of both interfacial abruptness and compositional variation, provided samples of constant thickness could be prepared.

Spot diffraction patterns recorded with the beam in the plane of the interfaces show superlattice reflections as expected (figure 3), indicating the high quality of the superlattice. We plan to collect these around $100 \mathrm{~K}$, where a peak is seen in magnetization ([3], fig 3(f) ) to identify any associated structural changes. A quantititive analysis of digital superlattice reflections by QCBED [4], in order to map out the charge-ordering in real-space, is complicated by the large number of atoms involved in the supercell, and the small Bragg angles, causing overlap of orders. This may be addressed by modelling all but the few atoms at the electronically unique interface, and by recording Kossel-like diffraction patterns, in which a large overlap of orders is permitted, and simulations performed for those experimental conditions. The use of incoherently superimposed orders is preferable, since it renders the intensity distribution insensitive to the location of the probe $[5,6]$.

References

[1] A. B. Shah et al., Phys. Rev. B. 82 (2010) 155112.

[2] J. Spence, Ch 77 in "Dislocations in Solids" Vol 13. Nabarro and Hirth. North-Holland. N.Y.

[3] B. B. Nelson-Cheeseman et al., Appl. Phys. Lett. In press. (2011).

[4] J. M. Zuo. For a review, see Rep Prog Phys. 67, 2053 (2004).

[5] J. Spence and J. Zuo. "Electron Microdiffraction" Plenum. 1992.

[6] Supported by DOE award DE-FG03-02ER45996 and collaboration with J.M.Zuo. 


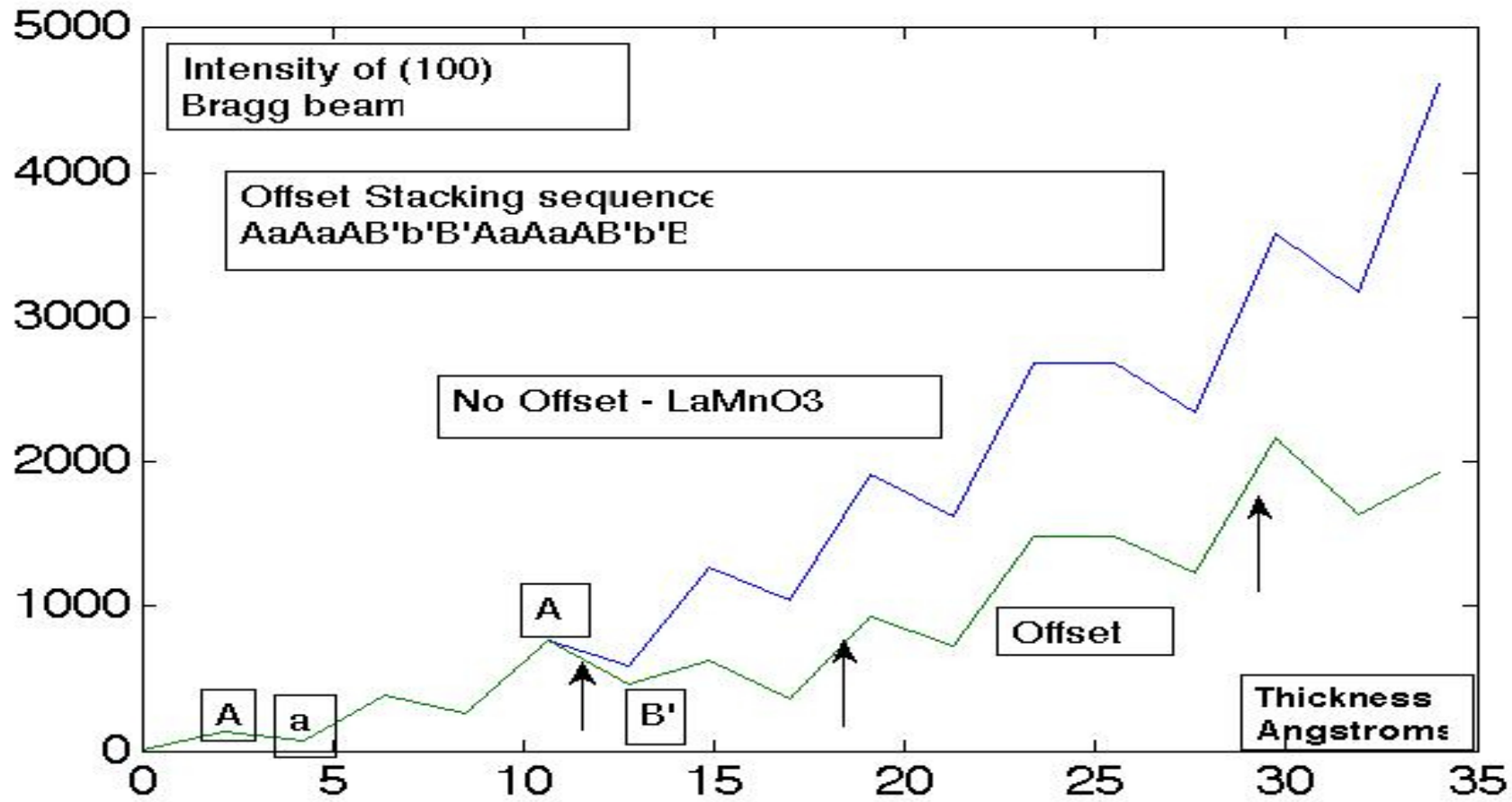

Figure 1. Intensity of (100) beam vs thickness with [001] incident beam for two stacking sequences.
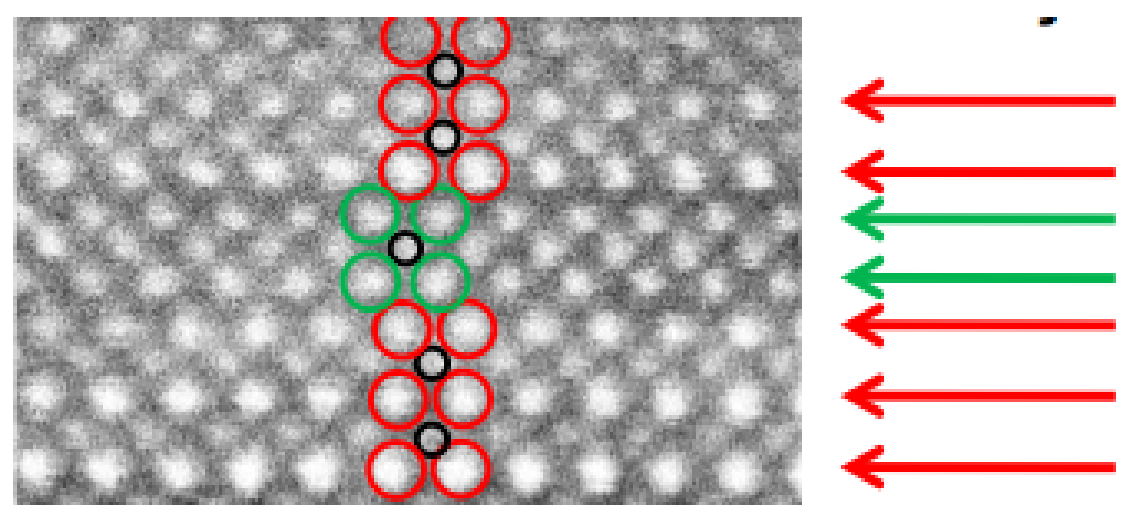

\section{LaO layers}

Offset SrO layers

\section{LaO layers}

Figure 2. $200 \mathrm{kV}$ ADF STEM image of perovskite digital superlattice from ref [3] (sample 0148) showing preliminary layer identification based on STEM contrast. [010] into page, [100] across.

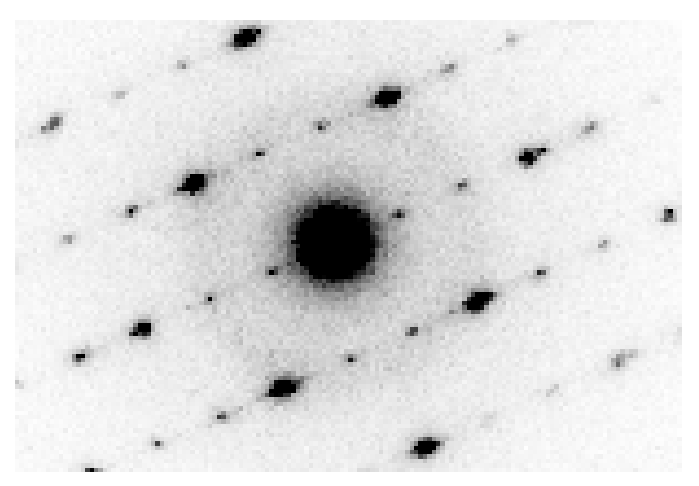

Figure 3. Transmission electron diffraction pattern at $200 \mathrm{kV}$ down [010] from sample in figure 2. 